\title{
COMPORTAMIENTO TRIBOLÓGICO DE UN ACERO INOXIDABLE AUSTENÍTICO EN TRIBÓMETRO BOLA SOBRE ANILLO
}

\section{Tribological Behavior of an Austenitic STAINLESS STEEL IN A BALL ON RING TRIBOMETER}

\author{
J. E. Romero C. ${ }^{1, *}$
}

\section{Resumen}

Se muestra la investigación realizada para la caracterización del comportamiento ante la fricción y desgaste de un acero inoxidable austenítico. Se realizaron ensayos en un tribómetro tipo bola sobre anillo con un diseño experimental basado en un arreglo de Taguchi. El material evaluado fue el acero inoxidable AISI 304 y como contraparte se usó el acero AISI 52100. A fin de estudiar la respuesta tribológica, los parámetros (y sus niveles) seleccionados fueron: velocidad de deslizamiento $(0,9 \mathrm{~m} / \mathrm{s}$ y $2 \mathrm{~m} / \mathrm{s})$, carga normal $(9 \mathrm{~N}$, $19 \mathrm{~N}$ y $29 \mathrm{~N})$ y distancias de deslizamiento $(500 \mathrm{~m}$, $1000 \mathrm{~m}$ y $2000 \mathrm{~m}$ ). Para las condiciones estudiadas, el coeficiente de fricción resultó en valores promedio entre 0,3227 a 0,9674, donde la carga normal fue el parámetro más influyente. Se obtuvieron valores del coeficiente de desgaste promedio en el intervalo desde 0,000860 a 0,003191 . El factor que más afecta el coeficiente de desgaste es la distancia de deslizamiento. El régimen de desgaste se identificó como moderado a severo. El mecanismo de desgaste para el AISI 304 en las condiciones estudiadas fue de adhesión.

Palabras clave: acero inoxidable austenítico, fricción por deslizamiento, desgaste adhesivo, método Taguchi, tribología.

\section{Abstract}

The research conducted for the characterization of the friction and wear behavior of an austenitic stainless steel is shown. Tests were performed on a ball-onring tribometer with an experimental design based on a Taguchi array. The evaluated material was AISI 304 stainless steel and AISI 52100 steel was used as a counterpart. The parameters (and their levels) selected in order to study the tribological response were: slip velocity $(0.9 \mathrm{~m} / \mathrm{s}$ and $2 \mathrm{~m} / \mathrm{s})$, normal load $(9 \mathrm{~N}, 19 \mathrm{~N}$ and $29 \mathrm{~N})$ and sliding distance $(500 \mathrm{~m}$, $1000 \mathrm{~m}$ and $2000 \mathrm{~m}$ ). For the studied conditions, the coefficient of friction resulted in average values between 0.3227 and 0.9674 , where the normal load was the most influential parameter. The average values of the coefficient of wear were obtained in the range from 0.000860 to 0.003191 . The factor that most affects the coefficient of wear is the slip distance. The wear regime was identified as moderate to severe. The wear mechanism for AISI 304 under the studied conditions was adhesion.

Keywords: austenitic stainless steel, sliding friction, adhesive wear, Taguchi method, tribology.

\footnotetext{
$\overline{1, *}$ Centro de Investigación en Materiales (CIM), Facultad de Ingeniería de la Universidad de Carabobo, Valencia, Venezuela. Autor para correspondencia: joromer@uc.edu.ve. (D) http://orcid.org/0000-0002-3759-8339

Recibido: 10-05-2017, aprobado tras revisión: 19-06-2017

Forma sugerida de citación: Romero, J. (2017). «Comportamiento tribológico de un acero inoxidable austenítico en tribómetro bola sobre anillo». INGENIUS. N. ${ }^{\circ} 18$, (julio-diciembre). pp. 64-72. ISSN: 1390-650X.
} 


\section{Introducción}

Los aceros inoxidables austeníticos son ampliamente usados en implantes biomédicos y en elementos de máquinas de la industria de alimentos y petroquímica, gracias a su alta resistencia a la corrosión.

Investigaciones previas han sido realizadas en tribómetros tipo espiga sobre disco a diferentes niveles cargas, velocidades y distancias de deslizamiento [1-3]. Sin embargo, aún no se conocen suficientemente algunas de sus propiedades o comportamientos ante las solicitaciones tribológicas, empleando un tribómetro tipo bola sobre anillo, siendo esta necesaria para desarrollar diseños de dispositivos óptimos en su durabilidad bajo condiciones de contacto no concordante.

El comportamiento friccional es afectado por una amplia lista de factores, como por ejemplo, la cinemática de las superficies en contacto y la aplicación externa de carga o el desplazamiento, lo que indica que el coeficiente de fricción no es una propiedad del material [4]. Para la comprensión del comportamiento friccional de los materiales se han planteado distintas teorías: los modelos de adhesión, de energía de superficie, de deformación plástica, de rugosidad, combinado adhesión-surcado-rugosidad y el de deformaciónsurcado-adhesión [4]. Este último, demuestra una mejor correlación entre el coeficiente de fricción teórico y el experimental [5].

La fricción se considera como la fuerza de resistencia tangencial en la frontera común entre dos cuerpos cuando, bajo la acción de una fuerza externa, un cuerpo se mueve o tiende a desplazarse con respecto a la superficie de la otra. Se distinguen principalmente dos clases de fricción, por deslizamiento y por rodamiento. La fricción por rodamiento implica a las superficies no concordantes, mientras que las superficies correspondientes al deslizamiento son concordantes. La razón entre la fuerza tangencial, $F$, y la carga normal aplicada, $W$, se conoce como el coeficiente de fricción $(\mathrm{CoF})$ y es $\mu=F / W$.

El desgaste se puede considerar como la pérdida continua indeseable de material de una o ambas superficies del apareamiento de elementos tribológicos debido al movimiento relativo de las superficies. El desgaste es determinado por las variables de funcionamiento, los tipos de materiales y la superficie involucrada, así como los factores ambientales. El desgaste puede ir acompañado de transferencia de material de una superficie a la otra. Los mecanismos de desgaste más comunes incluyen la adhesión, abrasión, fatiga superficial, corrosión (por frotamiento) y erosión [6]. El desgaste por adhesión es más frecuente y menos pronosticable.

Por lo general, el desgaste es inversamente proporcional a la dureza. Usualmente, el volumen de desgaste es independiente de la velocidad de deslizamiento y se expresa por la ecuación de Archard [7] como $V=K_{a} W L / H$, donde $V$, volumen de desgaste;
$W$, fuerza normal; $L$, distancia del deslizamiento y $H$ es la dureza de penetración del más blando de los dos materiales. El factor $K_{a}$ es el coeficiente de desgaste adimensional y es una característica del sistema deslizante en función de los materiales usados, las condiciones de operación y también de la lubricación y el lubricante.

En el presente artículo se reportan los resultados de la caracterización realizada de la fricción y el desgaste por deslizamiento en especímenes de un acero inoxidable austenítico tipo AISI 304 sin tratamiento térmico en aire, sin lubricación y a temperatura, presión y humedad normal de un laboratorio en un tribómetro tipo bola sobre anillo.

\section{Materiales y métodos}

En esta sección se describe brevemente el material de las muestras de prueba, la configuración experimental y las técnicas utilizadas para medir las variables seleccionadas.

\subsection{Preparación de las muestras}

Las probetas del acero AISI 304 se obtuvieron a partir del maquinado en torno de una barra maciza de $32 \mathrm{~mm}$ de diámetro externo, obteniéndose los anillos de $30 \mathrm{~mm}$ de diámetro externo, $8 \mathrm{~mm}$ de espesor y $10 \mathrm{~mm}$ de ancho.

Las bolas utilizadas fueron de acero AISI 52100 de $6 \mathrm{~mm}$ de diámetro, obtenidas a partir de cojinetes disponibles en el comercio. Se seleccionó dicho material como contraparte por su elevada dureza (aproximadamente cuatro veces más duro que el acero en estudio). Se empleó una bola diferente para cada muestra. En cuanto a la preparación de estas se les realizaron una limpieza con acetona y alcohol en un baño ultrasónico, con la finalidad de eliminar en lo posible todo residuo en la superficie.

\subsection{Ensayo en tribómetro bola sobre anillo}

Los ensayos de fricción y desgaste por deslizamiento se llevaron a cabo en un tribómetro tipo bola sobre anillo que se encuentra disponible en el Laboratorio de Predicciones de la Escuela de Ingeniería Mecánica de la Universidad de Carabobo, el cual es un desarrollo propio [8-10], y su esquema se muestra en la Figura 1. En este tribómetro se simula el comportamiento tribológico conforme a ASTM G77 [11], modificada para el contacto bola sobe anillo, realizándose la captación de los datos de fricción con un sistema automatizado, mediante la medición indirecta del torque de reacción a través de una celda de carga, visualizándose la información en un programa desarrollado para este fin.

Los parámetros que se pueden controlar en el tribómetro descrito son la carga normal, velocidad 
de deslizamiento y distancia o tiempo de duración del ensayo. En cuanto al movimiento, en esta máquina el anillo de prueba se instala sobre el eje del equipo, por lo cual este es el elemento móvil (ver Figura 1b), por su parte la bola se coloca en una portaesfera directamente en la barra de carga, por lo que permanece estacionaria, resultando un movimiento giratorio de contacto lineal no concordante.

Los datos referentes a la fricción, se obtuvieron a partir del sistema automático de adquisición de datos y el programa desarrollado para ambiente Windows, el cual registró la velocidad de giro, la carga normal, la fuerza de roce, el tiempo de ensayo y el coeficiente de roce o fricción, entre otros parámetros, a medida que se desarrolló cada experimento.

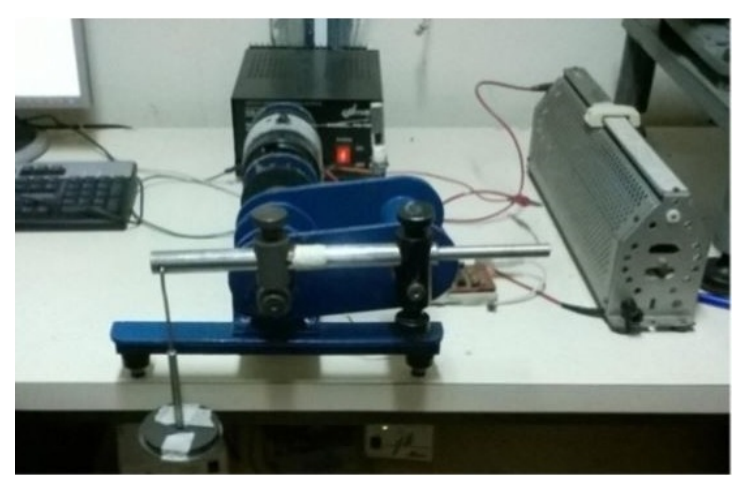

(a)

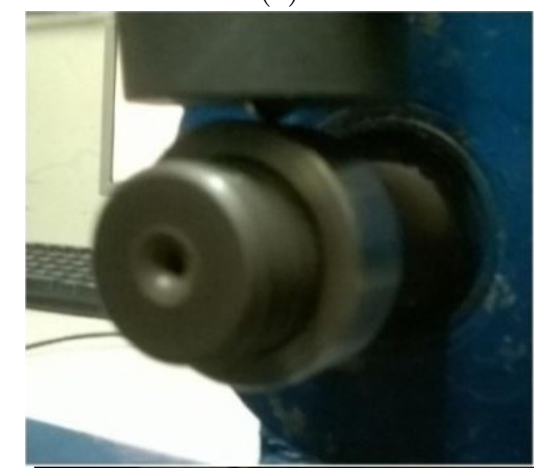

(b)

Figura 1. Tribómetro tipo bola sobre anillo (a) arreglo general del dispositivo (b) detalle de la geometría del contacto

\subsection{Diseño experimental}

La matriz de ensayo se planificó en función de evaluar los tres parámetros antes mencionados: carga normal, velocidad de deslizamiento y distancia para una aplicación biomédica, y considerando las limitaciones del equipo de ensayo, que puede permitir la comparación con otras investigaciones. En cuanto a las pruebas, estas se realizaron en condiciones de lubricación seca y ambiente normales en laboratorio.

Se desarrolló un diseño experimental basado en la metodología de arreglos ortogonales de Taguchi (DET), por la limitación en la cantidad de material. El DET permitió optimizar las probetas disponibles y el análisis de la razón señal a ruido, $S / N$, determinar la influencia en los resultados de los parámetros seleccionados. El procedimiento general de la aplicación de la metodología DET se encuentra disponible en [12]. $\mathrm{Al}$ aplicar esta metodología DET tomando como base los parámetros y niveles de ensayo, se obtuvo la matriz ortogonal L9, la cual se presenta en la Tabla 1. Cada ensayo se realizó con una repetición para conocer la variabilidad en los parámetros seleccionados.

Tabla 1. Matriz de ensayos DET

\begin{tabular}{cccc}
\hline Experimento & $\begin{array}{c}\text { Carga } \\
(\mathbf{N})\end{array}$ & $\begin{array}{c}\text { Velocidad } \\
(\mathbf{m} / \mathbf{s})\end{array}$ & $\begin{array}{c}\text { Distancia } \\
(\mathbf{m})\end{array}$ \\
\hline 1 & 9 & 0,9 & 500 \\
2 & 9 & 2 & 1000 \\
3 & 9 & 2 & 2000 \\
4 & 19 & 0,9 & 1000 \\
5 & 19 & 2 & 2000 \\
6 & 19 & 0,9 & 500 \\
7 & 29 & 0,9 & 2000 \\
8 & 29 & 2 & 500 \\
9 & 29 & 2 & 1000 \\
\hline
\end{tabular}

\subsection{Análisis estadístico de los datos}

Una vez ejecutada la experimentación prevista en el diseño y obtenidos los resultados para cada ensayo realizado, estos pueden ser utilizados para determinar el efecto de los parámetros y niveles.

Para determinar su influencia, DET cuenta con el análisis del cociente o razón $S / N$ [12]. La razón $S / N$ combina tanto el nivel medio (señal) de la característica y su variación en torno a esta media (ruido). Tres funciones de pérdida de la razón $\mathrm{S} / \mathrm{N}$ están disponibles dependiendo del tipo de característica o variable de respuesta: (1) «entre más pequeña mejor», la variable no excederá cierto valor máximo, (2) «entre más grande mejor», la variable no será mayor a un valor mínimo y (3) «nominal es lo mejor», la variable deberá tener un valor específico. De estas funciones «entre más pequeña es mejor» es la más adecuada para describir el comportamiento tribológico, donde la meta es minimizar la respuesta y por lo tanto, la seleccionada en esta investigación para identificar el parámetro más influyente en la fricción y el desgaste por deslizamiento en seco del acero evaluado.

La razón $S / N$, «entre más pequeña mejor» para la característica o variable de respuesta en estudio, es calculada mediante la transformación logarítmica de la función de pérdida de Taguchi 12, como se describe en la ecuación 1.

$$
S / N=-10 \log \frac{\sum y_{i}^{2}}{n}
$$


Donde $\mathrm{n}$ es el número de observaciones y $y_{i}$ son los datos observados.

Una vez calculados el número $S / N$ para cada parámetro y nivel, se calcula el valor de rango $\Delta\left(S / N_{\text {mayor }}-S / N_{\text {menor }}\right.$ del $\left.S / N\right)$ de cada parámetro. A medida que este $\Delta$ (delta) sea mayor, mayor será la influencia del parámetro en el desempeño en evaluación.

Adicionalmente, se realizó una interpretación de los resultados experimentales por análisis de promedio y análisis de la varianza (Anova). El análisis Anova generalmente se realiza sobre resultados experimentales con el fin de identificar los parámetros de control que son estadísticamente significativos [13].

\subsection{Determinación coeficiente de desgaste y mecanismo de desgaste}

Una vez realizados los experimentos de desgaste adherente se procedió a calcular el volumen de desgaste sufrido por las probetas, siguiendo la norma ASTM G77, modificada para la geometría bola sobre anillo [9], a partir de la medición del ancho y profundidad de la huella, mediante la utilización de un proyector comparador de perfiles con precisión de $1 \mu \mathrm{m}$.

Finalmente, para determinar el régimen y mecanismo del desgaste se realizó una inspección cualitativa de la condición final de las probetas ensayadas por inspección visual y por microscopía óptica haciendo uso de un microscopio metalográfico con capacidad 501000x equipado con cámara digital para captura de imagen.

\section{Resultados y discusión}

En esta sección se presentan los resultados obtenidos del coeficiente de fricción CoF y el coeficiente de desgaste $K_{a}$, a partir del ensayo tribológico. Se ejecutó la matriz de experimentos en el acero inoxidable AISI 304 con la metodología descrita en la sección anterior.

\subsection{Análisis del comportamiento a la fricción por deslizamiento}

Mediante la captura del sistema de adquisición de datos, el tribómetro arrojó los valores del CoF para las condiciones evaluadas en intervalos de tiempo o distancias aproximadamente regulares. En la Figura 2 se presentan una serie de curvas de los resultados del $\mathrm{CoF}$ en función de la distancia ensayada, cada gráfico muestra en línea continua, el valor promedio de las réplicas de cada experimento.

Al observar los gráficos (a) y (b) de la Figura 2 del comportamiento general de la $\mathrm{CoF}$ del acero estudiado, no se apreció un periodo estable definido, sino una serie de periodos de transición a lo largo del recorrido, ya reportadas en otras investigaciones [1]; esto se atribuye a las velocidades seleccionadas (mayor a $0,8 \mathrm{~m} / \mathrm{s}$ ), a la condición de superficie (baja rugosidad), a la severidad del contacto en la interface (un contacto lineal) y al mecanismo de desgaste predominante (adhesión).

Por consiguiente, se consideró un régimen moderadamente estable, resolviendo tomar un promedio de todos los datos obtenidos a partir de un recorrido de $250 \mathrm{~m}$ como la respuesta de $\mathrm{CoF}$ reportado para el acero evaluado. Los valores del CoF promedio de los experimentos resultaron en el intervalo 0,3227 a 0,9674 para las condiciones evaluadas.

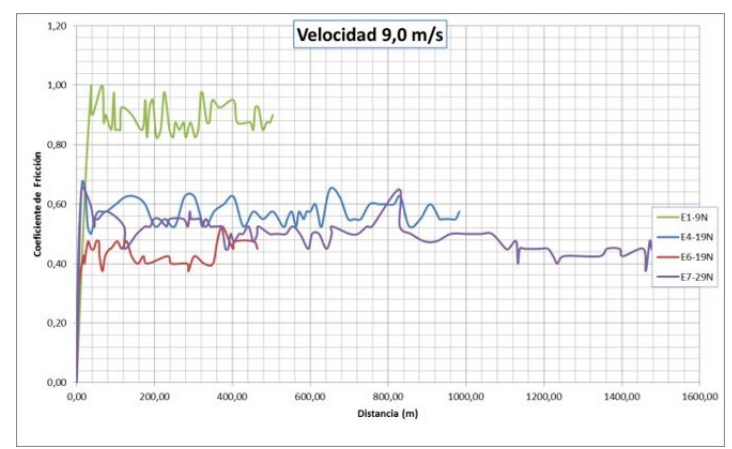

(a)

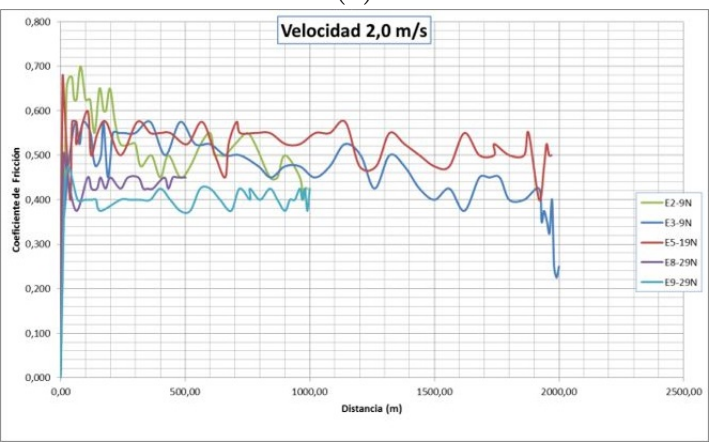

(b)

Figura 2. Resultados de CoF de cada experimento en función de la distancia de deslizamiento para (a) el nivel bajo, (b) el nivel alto de velocidad de deslizamiento.

En la Figura 3 se muestra la respuesta de fricción por deslizamiento para el acero AISI 304 considerando la carga, la velocidad o la distancia en sus efectos principales.

De la Figura 3, al analizar el efecto de la carga normal evaluada considerando la velocidad como fija se observó que el CoF disminuye a medida que la carga aumenta, es decir, la fuerza de fricción es proporcional a la carga normal, como se espera para la fricción seca de acuerdo con la teoría (1. ${ }^{\mathrm{a}}$ ley de Amontons-Coulomb). Los valores promedio del CoF para $9 \mathrm{~N}, 19 \mathrm{~N}$ y $29 \mathrm{~N}$ son $0,605876,0,512302$ y 0,445050 , respectivamente.

De igual manera, al examinar la Figura 3 se reseña el comportamiento de la fricción respecto a la velocidad de deslizamiento. Se puede destacar que para todos los experimentos realizados con el acero estudiado se obtuvo que el CoF disminuye con el aumento de la 
velocidad tangencial, siendo entonces esta respuesta una excepción a la $3 .^{\text {a }}$ ley de Coulomb, que establece que la fuerza de fricción es independiente de la velocidad. Para la velocidad de deslizamiento de $0,9 \mathrm{~m} / \mathrm{s} \mathrm{y}$ $2 \mathrm{~m} / \mathrm{s}$, se tiene un CoF promedio de 0,599155 y 0,458613 , respectivamente.

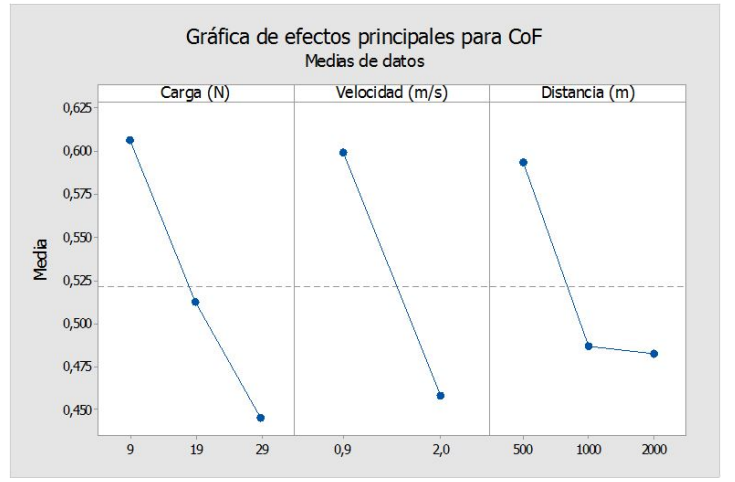

Figura 3. Respuesta de CoF promedio en función de la carga normal, la velocidad de deslizamiento y distancia de deslizamiento.

\subsection{Análisis del comportamiento del desgaste por deslizamiento}

En la Tabla 2 se listan los resultados de la evaluación del comportamiento de desgaste por deslizamiento del acero AISI 304 para las condiciones propuestas, reportando los datos para el volumen de desgaste y el coeficiente de desgaste $\left(K_{a}\right)$ para cada experimento. Todos los experimentos se muestran con dos repeticiones, señaladas con los subíndices a y b.

Tabla 2. Comportamiento de desgaste para el acero AISI 304

\begin{tabular}{ccc}
\hline Experimento & $\begin{array}{c}\text { Volumen de } \\
\text { desgaste }\left[\mathbf{m m}^{3}\right]\end{array}$ & $\begin{array}{c}\text { Coeficiente } \\
\text { de desgaste }\end{array}$ \\
\hline 1a & 7,27925576 & 0,003190995 \\
1b & 4,814311325 & 0,002110441 \\
2a & 8,910025457 & 0,001952936 \\
2b & 8,032251586 & 0,001760542 \\
3a & 7,843362199 & 0,00085957 \\
3b & 8,682818572 & 0,000951568 \\
4a & 10,43162011 & 0,001076686 \\
4b & 12,88165537 & 0,001329563 \\
5a & 17,86089144 & 0,000921744 \\
5b & 17,16137769 & 0,000885645 \\
6a & 13,42703445 & 0,002771708 \\
6b & 13,69781763 & 0,002827605 \\
7a & 29,63030803 & 0,001333356 \\
7b & 40,26323979 & 0,001811835 \\
8a & 20,93583908 & 0,002826322 \\
8b & 19,19093329 & 0,002590761 \\
9a & 44,02116044 & 0,002971411 \\
9b & 21,26842887 & 0,001435611 \\
\hline
\end{tabular}

De la Tabla 2 se observa que los valores para el volumen de desgaste resultaron en un intervalo 4,8143 a $44,0212 \mathrm{~mm}^{3}$ y los valores del coeficiente de desgaste en el intervalo desde 0,000860 hasta 0,003191.

En las Figuras 4 y 5 se muestran el comportamiento del volumen y coeficiente de desgaste promedio como una función de los parámetros ensayados y donde se señala los efectos sobre esta variable de la velocidad deslizamiento, la carga normal y la distancia de deslizamiento.

En la Figura 4 se interpreta el efecto de la distancia de deslizamiento, la carga normal y la velocidad de deslizamiento sobre el volumen de desgaste. Se observa que el volumen de desgaste se incrementa con el incremento de la distancia de deslizamiento e igual comportamiento para la variación de la carga normal; esta proporcionalidad es descrita por la ecuación Archard.

Adicionalmente, se aprecia que el volumen de desgaste crece con el incremento de la velocidad de deslizamiento, pero con efecto menor que la carga y la distancia.

En la Figura 5 se observa que el coeficiente de desgaste decrece con el incremento de la distancia de deslizamiento. Los valores promedio del Ka para $500 \mathrm{~m}, 1000 \mathrm{~m}$ y $2000 \mathrm{~m}$ son $0,002720,0,001754$ y 0,001127 , respectivamente.

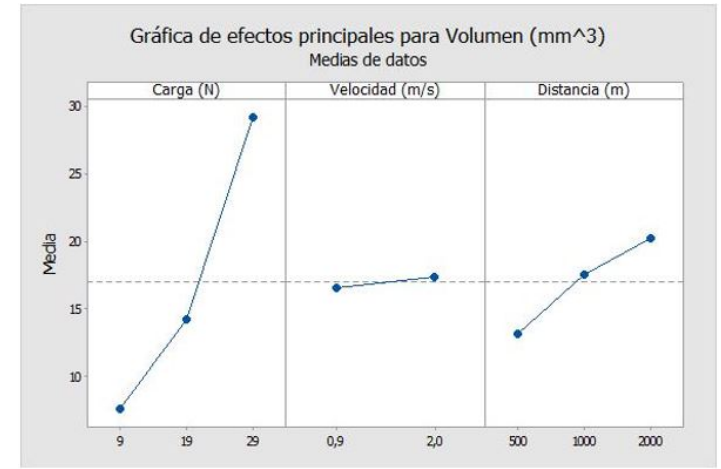

Figura 4. Respuesta de volumen de desgaste promedio en función de la carga normal, la velocidad de deslizamiento y distancia de deslizamiento.

Respecto al efecto de la velocidad de deslizamiento sobre el coeficiente de desgaste, este disminuye al incrementar el nivel de la velocidad. Para la velocidad de deslizamiento de $0,9 \mathrm{~m} / \mathrm{s}$ y $2 \mathrm{~m} / \mathrm{s}$, se tiene un Ka promedio de 0,002057 y 0,001716, respectivamente.

Por último, se nota efecto de la carga normal; en general el coeficiente de desgaste aumenta con el aumento de la carga, siendo los valores promedios del Ka para $9 \mathrm{~N}, 19 \mathrm{~N}$ y $29 \mathrm{~N}, 0,001804,0,001635$ y 0,002162 , respectivamente, para el acero en estudio.

\subsection{Análisis de la varianza}

Un análisis de varianza, se realizó mediante la aplicación Minitab®, programa específicamente empleado para el diseño y análisis experimental. 


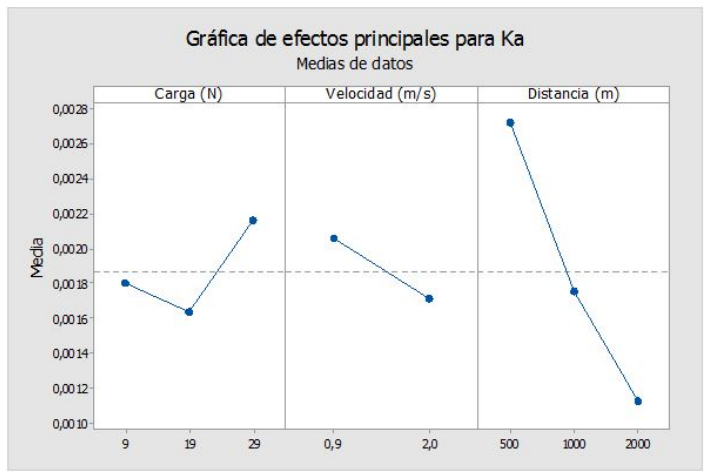

Figura 5. Respuesta de coeficiente de desgaste promedio en función de la carga normal, la velocidad de deslizamiento y distancia de deslizamiento.

$\mathrm{Al}$ interpretar la información del Anova para el coeficiente de fricción, mostrado en la Tabla 3, se obtuvo que en los experimentos realizados los parámetros carga normal y velocidad de deslizamiento son estadísticamente significativos al reportar el valor de la probabilidad $\mathrm{P}$, menor que 0,05 (nivel de significancia de $95 \%$ ).

En la Tabla 4 se muestra el Anova para el coeficiente de desgaste; resulta que en los experimentos realizados solo el parámetro de distancia de deslizamiento es estadísticamente significativo.

Tabla 3. Análisis Anova para CoF del AISI 304

\begin{tabular}{lccccc}
\hline Fuente & GL & SC Ajust. & MC Ajust. & Valor F & Valor p \\
\hline $\mathrm{A}\left(^{*}\right)$ & 2 & 0,105384 & 0,052692 & 6,8 & 0,014 \\
$\mathrm{~B}\left(^{* *}\right)$ & 1 & 0,082916 & 0,082916 & 10,71 & 0,008 \\
$\mathrm{C}\left(^{* *}\right)$ & 2 & 0,005493 & 0,002747 & 0,35 & 0,71 \\
Error & 10 & 0,077443 & 0,007744 & & \\
Total & 17 & 0,407783 & & & \\
\hline$\left(^{*}\right)$ Carga $(\mathrm{N})$ & & & & \\
$\left(^{* *}\right)$ Velocidad (m/s) & & & \\
$(* * *)$ &
\end{tabular}

Tabla 4. Análisis Anova para CoF del AISI 304

\begin{tabular}{lccccc}
\hline Fuente & GL & SC Ajust. & MC Ajust. & Valor F & Valor p \\
\hline A $\left(^{*}\right)$ & 2 & 0,000001 & 0,000000 & 1,89 & 0,201 \\
$\mathrm{~B}\left(^{* *}\right)$ & 1 & 0,000000 & 0,000000 & 0,00 & 0,962 \\
$\mathrm{C}\left(^{* * *}\right)$ & 2 & 0,000003 & 0,000002 & 6,08 & 0,019 \\
Error & 10 & 0,000003 & 0,000000 & & \\
Total & 17 & 0,000011 & & & \\
\hline$\left(^{*}\right)$ Carga $(\mathrm{N})$ & & & & \\
$\left(^{* *}\right)$ Velocidad (m/s) & & & \\
$\left(^{* * *}\right)$ Distancia $(\mathrm{m})$ &
\end{tabular}

\subsection{Razón señal a ruido}

Como se expuso en la sección anterior, la metodología Taguchi presenta un análisis que permite identificar aquellos parámetros que tienen mayor influencia en los resultados de fricción y desgaste de manera más eficiente. Los valores $S / N$ obtenidos con la aplicación Minitab®, para los factores y niveles evaluados se muestran en la Figura 6 y la Figura 7 (considerando que el más pequeño es mejor).

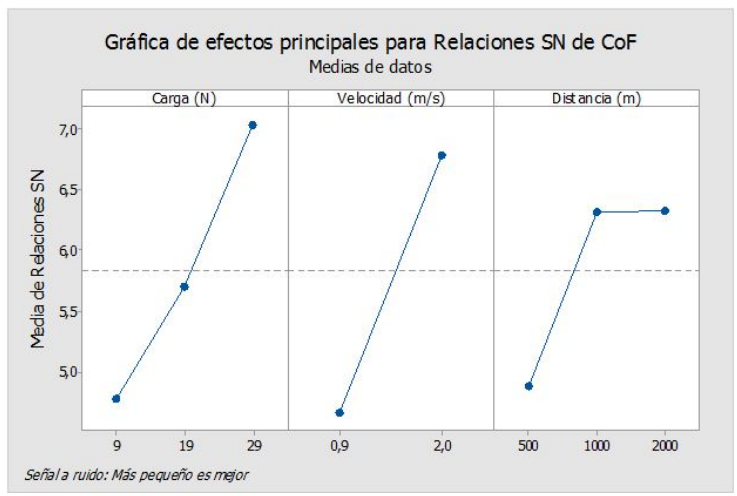

Figura 6. Gráficas de efectos para la variable señal a ruido (S/N), de CoF del AISI 304.

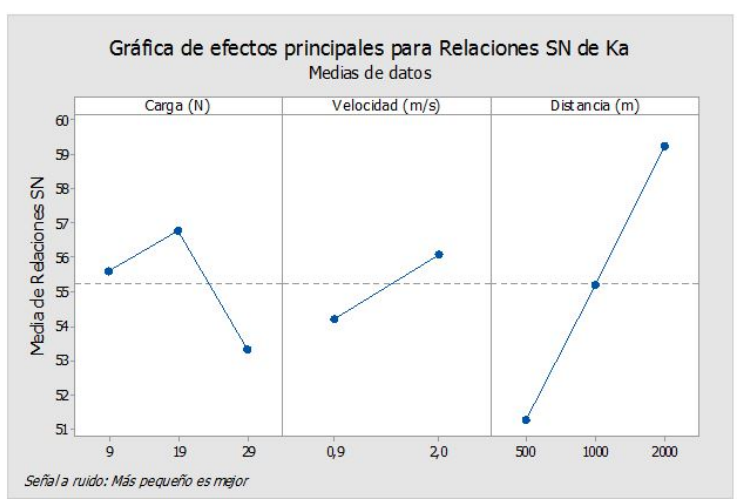

Figura 7. Gráficas de efectos para la variable señal a ruido $(\mathrm{S} / \mathrm{N})$, de Ka del AISI 304

De las gráficas de la Figura 6, se observa que los parámetros carga y velocidad son los que más afectan a la razón $S / N$, es decir, esto parámetros, son los de mayor influencia sobre la variación del CoF del acero AISI 304.

De la Figura 7 se interpreta la respuesta para relaciones de señal a ruido $S / N$ para el coeficiente de desgaste, resultando que la distancia de deslizamiento es el parámetro con el mayor efecto sobre el coeficiente de desgaste, seguido por la carga normal y por último, la velocidad de deslizamiento, como el parámetro que tiene el menor efecto en el coeficiente de desgaste del acero AISI 304.

\subsection{Análisis por microscopía óptica}

En esta sección se resume el estudio realizado por microscopía óptica (MO) a la huella marcada en la circunferencia del anillo de acero AISI 304.

Un análisis general de la evolución del daño para el rango de parámetros evaluados, indica que la condición de desgaste por deslizamiento del acero AISI 304 se interpretó como de moderado a severo, al estar caracterizado por valores del coeficiente de desgaste mayores a $20 \times 10-6$ [14]. 
Al observar imágenes obtenidas mediante microscopía óptica de las huellas de desgaste ensayadas (Figuras 8-16) se aprecia las evidencias donde se destaca que el mecanismo de desgaste principal fue el desprendimiento de material por el proceso de soldadura en frío (adhesión).

En la micrografía del evento E1a se aprecia deformación plástica del material, así como también rayaduras, producto de partículas de material desprendido que no se pudo liberar, con un desgaste considerado moderado. Para el evento E1b se evidencia de igual manera rayaduras, se observó un cráter producto del desprendimiento de material por el proceso de soldadura en frío y un desgaste considerado moderado (ver Figura 8).

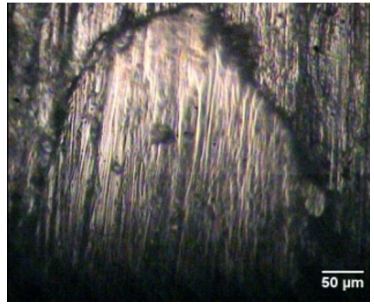

(a) Ensayo 1a

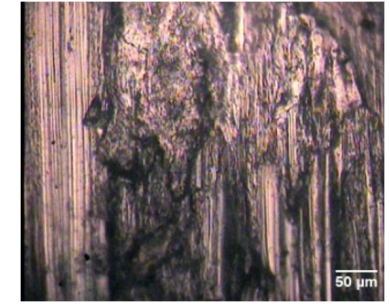

(b) Ensayo 1b
Figura 8. Micrografías obtenidas por microscopía óptica a 200x de la huella de desgaste del experimento 1 del acero AISI 304.

En el evento E2a la micrografía presentó rayaduras en la dirección del movimiento, grietas y un desgaste considerado severo. En el evento E2b se apreció de igual manera grietas, rayaduras y zonas con desprendimiento de material debido al proceso de soldadura en frío (ver Figura 9).

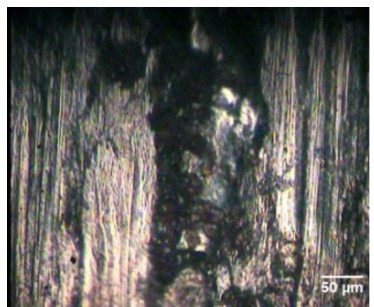

(a) Ensayo 2a

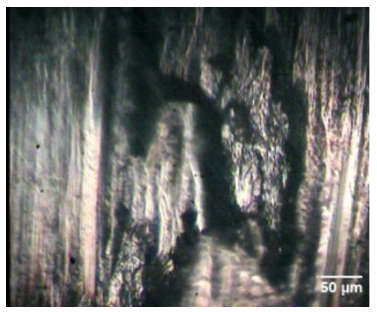

(b) Ensayo $2 \mathrm{~b}$
Figura 9. Micrografías obtenidas por microscopía óptica a 200x de la huella de desgaste del experimento 2 del acero AISI 304.

En el evento E3a se observó un desgaste severo, la aparición de un cráter que se podría considerar grande debido al proceso de soldadura en frío y un desgaste que se puede considerar severo. En el evento E3b se apreció un cráter de pequeña dimensión, rayaduras leves debido a la falta de liberación de material desprendido y un desgaste que se puede considerar moderado (ver Figura 10).

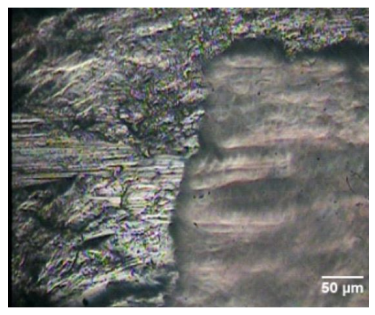

(a) Ensayo 3a

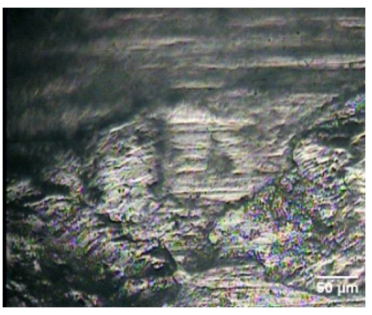

(b) Ensayo 3b
Figura 10. Micrografías obtenidas por microscopía óptica a 200x de la huella de desgaste del experimento 3 del acero AISI 304.

Para el evento E4a la micrografía mostró deformación plástica del material, grietas con un desgaste considerado severo. En el evento E4b se observaron rayaduras y una zona amplia con desprendimiento de material por el proceso de soldadura con un desgaste moderado (ver Figura 11).

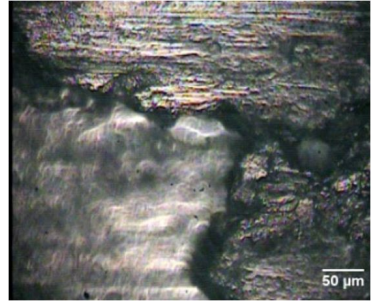

(a) Ensayo 4a

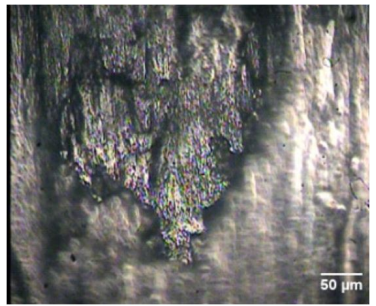

(b) Ensayo 4b
Figura 11. Micrografías obtenidas por microscopía óptica a 200x de la huella de desgaste del experimento 4 del acero AISI 304.

Al observar el evento E5a en la micrografía se percibió una grieta central y un desgaste moderado. Para el evento E5b se observó una serie de grietas de mayor tamaño con desprendimiento de material producto del proceso de soldadura en frío y un desgaste considerado moderado (ver Figura 12).

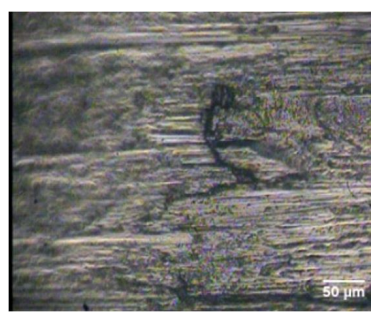

(a) Ensayo 5a

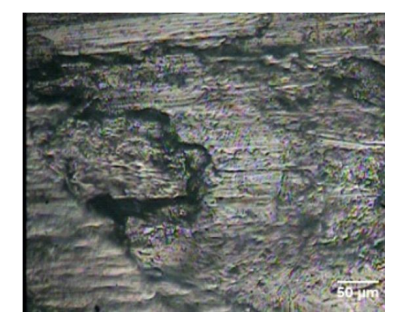

(b) Ensayo 5b
Figura 12. Micrografías obtenidas por microscopía óptica a 200x de la huella de desgaste del experimento 5 del acero AISI 304.

En el evento E6a la micrografía arrojó la presencia de un desgaste moderado, pequeñas grietas y cráteres de pequeña dimensión. En el evento E6b se observó gran cantidad de grietas con un cráter central de gran 
tamaño producto de la soldadura en frío y un desgaste considerado severo (ver Figura 13).

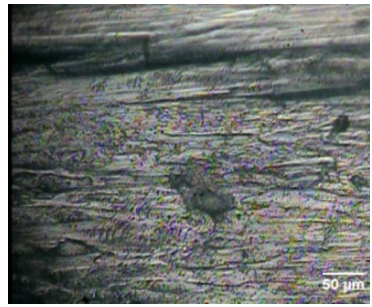

(a) Ensayo 6a

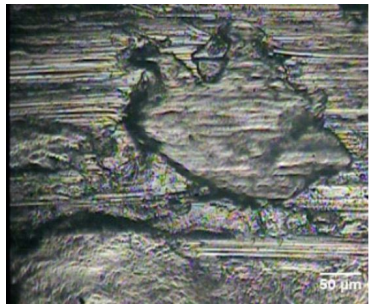

(b) Ensayo 6b
Figura 13. Micrografías obtenidas por microscopía óptica a 200x de la huella de desgaste del experimento 6 del acero AISI 304.

En el evento E7a la micrografía presentó un cráter de gran tamaño, producto del desprendimiento de material por el proceso de soldadura en frío, con ligeras rayaduras y un desgaste severo. Por su parte el evento E7b se apreciaron un número considerable de grietas y un cráter causado por el material debido al proceso de soldadura en frío (ver Figura 14).

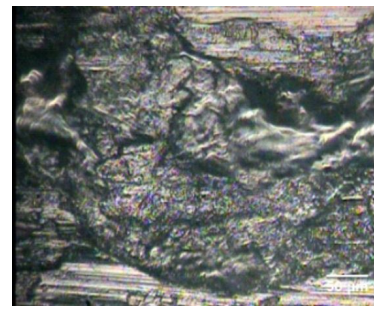

(a) Ensayo $7 \mathrm{a}$

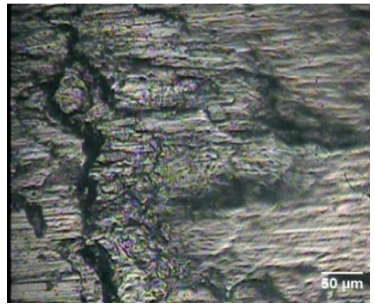

(b) Ensayo $7 \mathrm{~b}$
Figura 14. Micrografías obtenidas por microscopía óptica a 200x de la huella de desgaste del experimento 7 del acero AISI 304.

$\mathrm{Al}$ examinar el evento E8a en la micrografía se evidenció un desgaste moderado, con rayaduras, un cráter producto del proceso de soldadura en frío y grietas. En el evento E8b se observó un cráter de gran tamaño, grietas y un desgaste severo (ver Figura 15).

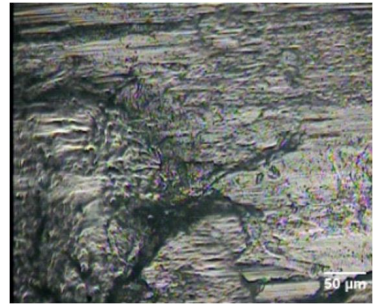

(a) Ensayo 8a

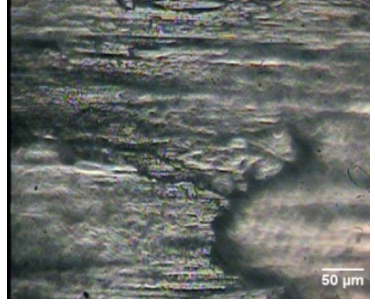

(b) Ensayo 8b
Figura 15. Micrografías obtenidas por microscopía óptica a 200x de la huella de desgaste del experimento 8 del acero AISI 304.

$\mathrm{Al}$ examinar el evento E9a la micrografía mostró una apreciable cantidad de rayaduras, con la aparición de un cráter producto del proceso de soldadura en frío y un desgaste considerado moderado. En el evento E9b se observó numerosas rayaduras con la presencia de un cráter producto de la soldadura y un desgaste considerado moderado (ver Figura 16).

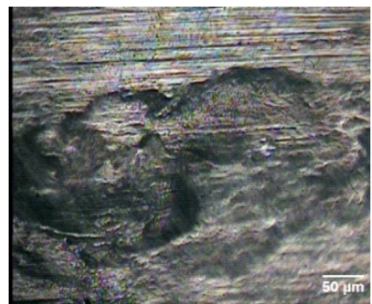

(a) Ensayo 9a

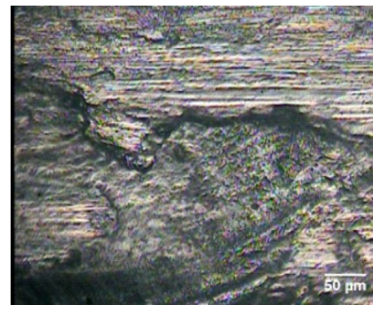

(b) Ensayo 9b
Figura 16. Micrografías obtenidas por microscopía óptica a 200x de la huella de desgaste del experimento 8 del acero AISI 304.

\section{Conclusiones}

Dado los resultados obtenidos de la caracterización tribológica del acero inoxidable AISI 304 se concluye lo siguiente: Para el coeficiente de fricción, la carga normal resultó ser el parámetro más influyente y la distancia de deslizamiento, el de menor influencia en los niveles estudiados. El parámetro que más afectó al coeficiente de desgaste del acero AISI 304 fue la distancia de deslizamiento. El régimen de desgaste se identificó como moderado a severo para las condiciones estudiadas. El mecanismo de desgaste principal presente en las muestras estudiadas fue el desprendimiento de material por el proceso de soldadura en frío (adhesión).

\section{Agradecimientos}

El autor desea expresar su especial agradecimiento a docentes, técnicos y auxiliares de investigación del Laboratorio de Materiales y del Laboratorio de Predicciones de la Escuela de Ingeniería Mecánica, UC, por su valiosa colaboración en la realización de las pruebas. Al Centro de Investigación de Materiales de la Facultad de Ingeniería, UC, por el financiamiento parcial de este proyecto.

\section{Referencias}

[1] G. Straffelini, A. Molinari, and D. Trabucco, "Sliding wear of austenitic and austenitic-ferritic stainless steels," Metallurgical and Materials Transactions A, vol. 33, no. 3, pp. 613-624, 2002. [Online]. Available: http://dx.doi.org/10.1007/s11661-002-0123-4

[2] M. Farias, R. Souza, A. Sinatora, and D. Tanaka, "The influence of applied load, 
sliding velocity and martensitic transformation on the unlubricated sliding wear of austenitic stainless steels," Wear, vol. 263, no. 1, pp. 773-781, 2007, 16th International Conference on Wear of Materials. [Online]. Available: http://dx.doi.org/10.1016/j.wear.2006.12.017

[3] M. A. Chowdhury, D. M. Nuruzzaman, and B. K. Roy, "Experimental investigation of friction coefficient and wear rate of stainless steel 304 sliding against smooth and rough mild steel counterfaces," Gazi University Journal of Science, vol. 26, no. 4, pp. 597-609, 2013. [Online]. Available: https://goo.gl/61XHDF

[4] N. Suh, Tribophysis. Prentice-Hall, 1986.

[5] N. P. Suh and H.-C. Sin, "The genesis of friction," Wear, vol. 69, no. 1, pp. 91-114, 1981. [Online]. Available: http: //dx.doi.org/10.1016/0043-1648(81)90315-X

[6] C. Kajdas, E. Wilusz, and S. Harvey, Encyclopedia of Tribology. Tribology series. Elsevier Science, 1990 .

[7] J. F. Archard, "Contact and rubbing of flat surfaces," Journal of Applied Physics, vol. 24, no. 8, pp. 981-988, 1953. [Online]. Available: http://dx.doi.org/10.1063/1.1721448
[8] K. Turkintong, "Diseño y fabricación de una máquina de ensayo de roce," Universidad de Carabobo, Venezuela, 1980.

[9] L. Medina and G. Peralta, "Evaluación de las propiedades tribológicas del acero dúplex," Universidad de Carabobo, Venezuela, 2010.

[10] C. Gloriet, "Desarrollo del sistema de medición y control de un tribómetro tipo bloque en anillo," Universidad de Carabobo, Venezuela, 2012.

[11] ASTM, ASTM Gr7-98 Standard Test Method for Ranking Resistance of Materials to Sliding Wear Using Block-on-Ring Wear Test, vol. 03.02, West Conshohocken, PA, ASTM International, ASTM Wear and Erosion; Metal Corrosion Std., 2004.

[12] R. Roy, A Primer on the Taguchi Method, 2nd ed. Society of Manufacturing Engineers, 2010.

[13] W. DeCoursey, "Statistics and probability for engineering applications," W. DeCoursey, Ed. Newnes, 2003. [Online]. Available: https://goo.gl/SJiKqm

[14] Y. Wang, T. Lei, and J. Liu, "Tribometallographic behavior of high carbon steels in dry sliding," Wear, vol. 231, no. 1, pp. 1-11, 1999. [Online]. Available: http: //dx.doi.org/10.1016/S0043-1648(99)00115-5 